\title{
An Evidence Combination Rule Based on New Weight Assignment Scheme
}

yucui wang ( $\sim 1540258530 @ q q . c o m$ )

Zhengzhou University https://orcid.org/0000-0002-8742-0459

Jian Wang

Zhengzhou University https://orcid.org/0000-0003-3279-9948

Mengjie Huang

Zhengzhou University

Minghui Wang

Zhengzhou University

\section{Research Article}

Keywords: D-S evidence theory, Evidence combination, Weight assignment, Conflicting evidence, Fuzzy evidence

Posted Date: January 5th, 2022

DOI: https://doi.org/10.21203/rs.3.rs-1024471/v1

License: (c) (1) This work is licensed under a Creative Commons Attribution 4.0 International License. Read Full License 


\title{
An evidence combination rule based on new weight
}

\section{assignment scheme}

\author{
Yu-Cui Wang ${ }^{1}$, Jian Wang ${ }^{1 *}$, Meng-Jie Huang ${ }^{1}$ and Ming-Hui Wang ${ }^{1}$ \\ ${ }^{1 *}$ School of Information Engineering of ZZU, Zhengzhou University, No. 100, Science \\ Avenue, Zhengzhou, 450001, Henan, China.
}

*Corresponding author(s). E-mail(s): iejwang@zzu.edu.cn;

\begin{abstract}
Conflicting evidence and fuzzy evidence have a significant impact on the results of evidence combination in the application of evidence theory. However, the existing weight assignment methods can hardly reflect the significant influence of fuzzy evidence on the combination results. Therefore, a new method for assigning evidence weights and the corresponding combination rule are proposed. The proposed weight assignment method strengthens the consideration of fuzzy evidence and introduces the Wasserstein distance to compute the clarity degree of evidence which is an important reference index for weight assignment in the proposed combination rule and can weaken the effect of ambiguous evidence effectively. In the experiments, it's firstly verified that the impact of fuzzy evidence on the combination results is significant; therefore it should be fully considered in the weight assignment process. Then, the proposed combination rule with new weight assignment method is tested on a set of numerical arithmetic and Iris datasets. Compared with four existing methods, the results show that the proposed method has higher decision accuracy, F1 score, better computational convergence, and more reliable fusion results as well.
\end{abstract}

Keywords: D-S evidence theory, Evidence combination, Weight assignment, Conflicting evidence, Fuzzy evidence

\section{Introduction}

Evidence theory (Dempster, 1967; Shafer, 1976) is an information fusion approach formally proposed by Dempster in 1967 and then further refined and popularized by his student Shafer, also known as DEMPSTER-SHAFER (D-S) evidence theory. DS evidence theory extends the basic event space in traditional probability theory into the power set space of basic events, and establishes the Basic Probability Assignment function (BPA) and evidence combination rule. Evidence theory has a stronger fusion ability in dealing with uncertain information and can make decisions on conflicting and fuzzy evidence without prior knowledge. 
It is closer to human thinking habit in solving the uncertainty measurement of multi-source information, and its reasoning mechanism is relatively simple. At present, evidence theory has been used in many fields, e. g., military command (Xu et al, 2018), target tracking (Gruyer et al, 2016), state recognition (Huang et al, 2018), image processing (Lian et al, 2019), fault diagnosis (Wang et al, 2019b; Zhang and Deng, 2020), intelligent decision making (Fei et al, 2019; Ma et al, 2019), and medical diagnosis (Zhou et al, 2020). However, in practical application, because the data comes from multiple information sources and is affected by uncertain environmental factors, there is usually highly uncertain evidence in the data sample. Uncertain evidence is mainly divided into conflict evidence (Zadeh, 1984, 1986) (the target that the evidence points to is obviously discordant) and fuzzy evidence Dubois and Prade (1985) (which does not clearly point to a clear target), as shown in Figure 1. If the evidence involved in the fusion contains high uncertainty, the D-S evidence combination rule may draw a conclusion contrary to the actual situation (Zadeh, 1984).

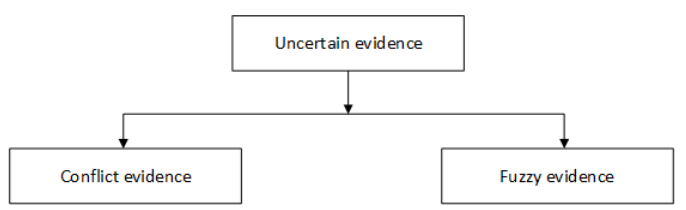

Fig. 1 Uncertain Evidence in Evidence Theory.

To solve the above defects in the evidence theory combination rule, researchers put forward many improvement methods to adapt to the existence of highly uncertain evidence and improve the accuracy of decision-making. The first method is to modify the classical Dempster combination rule framework. Such methods researchers believe that the normalization operation of the original combination rules is unreasonable, so it should be focused on how to allocate and resolve conflicts. These researches can be divided into the following categories according to their different ideas: conflict redistribution based on unified reliability function model (Yager, 1987; Deng and Shi, 2003), fusion rules based on set attribute relations (Xu et al, 2004), allocation method involving conflict between focal element based on local conflicts (Wang et al, 2001, 2019a) and combination rules based on open recognition framework (Smets, 1990; Xu et al, 2008). The second method is to correct evidence sources before combination. These researchers believe that the main reasons affecting the decision-making results are the conflicting and high-degree fuzziness of evidence, and the focus of this problem is how to determine the weight of evidence by the uncertainty measurement (Han et al, 2011). The specific process of this kind of method is to preprocess the evidence using weight firstly, and then combine the processed evidence according to the Dempster combination rule. There are also two branches of 
such kind of methods, i. e., the weighted average correction method (Murphy, 2000; Sun et al, 2020; Chen et al, 2021) and the discount coefficient method (Jousselme et al, 2001; Lefèvre and Elouedi, 2013; Song et al, 2014; Hu et al, 2016; Xue et al, 2021; Xu and Deng, 2018). Compared with modifying the combination rule framework, such method retains the excellent mathematical properties of D-S evidence theory, and more effectively solve the problems caused by conflicting evidence and fuzzy evidence in combination, therefore it has better practical application value. Obviously, the decision-making result of the second method is greatly influenced by the weight of evidence, so how to measure the uncertainty of evidence accurately and assign the weight reasonably is the focus and difficulty of such researches. At present, the uncertainty measurement (Xiao, 2021a) of evidence mainly involves the conflicting measurement (indicated by conflict degree or credibility degree) for conflicting evidence, and ambiguity measurement (indicated by ambiguity degree or clarity degree) for fuzzy evidence, as shown in Figure 2. Previous studies tend to pay more attention to the conflict of evidence, while ignore or underestimate fuzzy evidence's impact on decision-making results. However, the probability of fuzzy evidence is usually higher than that of conflicting evidence in real situation. Meanwhile, the impact of fuzzy evidence on the final decision is no less than that of conflicting evidence.

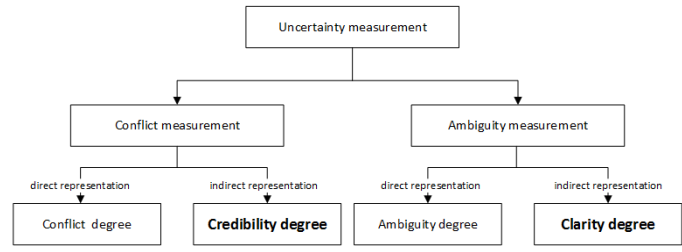

Fig. 2 Uncertainty measure in evidence theory

Therefore, in view of the shortcomings of existing schemes, by considering the characteristics of actual evidence, a new evidence weight assignment method and the combination rule based on it are presented in this paper. The method uses the Wasserstein distance to calculate the clarity degree of evidence, fusing the credibility degree of evidence based on the Jousselme distance and Sort-Factor to calculate the weight of evidence and realize the modification of evidence. Sequencely, the modified evidence is combined $N-1$ times by using the Dempster combination formula to get the final result.

The rest of this paper is assigned as follows. Section 2 introduces the related work. Section 3 describes the terms and formulas used in the paper. The proposed method is presented in Section 4. And in Section 5, the experimental results to compare the performance of the presented method with the other four methods are analyzed and summarized. At last, Section 6 summarizes and discusses our method and outlines the future research directions. 


\section{Relate work}

One of the main bases of evidence weight assignment is the conflict measurement to the evidence. Some scholars measure the evidence conflict by directly calculating the conflict degree of it. Wei (2011) introduced the K-L (KullbackLeibler) divergence and proposed a new method to combine conflict evidence. Similarly, Li et al (2014) used K-L divergence instead of the traditional D-S conflict coefficient $\mathrm{K}$ to characterize the conflict degree of evidence in the system, and optimized the scope of application and convergence performance of D-S evidence theory. But the K-L divergence does not satisfy the symmetry and Triangle inequality of the distance. Xiao (2020b) used a new reinforced belief divergence measure $(\mathrm{RB})$ to measure the difference between BPAs. Li et al (2020) and Fu et al (2021) introduced Hellinger distance and belief Coulomb force to measure the conflict degree of evidence, respectively. Some other scholars use the indirect method to measure the conflict degree of evidence, which is generally done by first measuring the similarity between evidence through a distance function and then converting it into credibility. Considering that different distance functions have different effects in describing the credibility of evidence, Lin et al (2016) proposed a combination method based on Mahalanobis distance function, however, Mahalanobis distance function requires computing the covariance of matrix, which is not suitable for large-scale data processing. Ye et al (2017) proposed a combination method based on the Lance distance function, but it did not take into account the ambiguity measure of evidence. Zhao et al (2013) used closeness degree to measure the credibility of evidence and Lei and Sheng (2021) optimized the credibility later. Jousselme et al (2001) and Zhang et al (2020) measured the credibility degree of evidence by calculating the Jousselme distance function, taking into account the number of elements in each hypothesis, which can effectively make use of the global information of each piece of evidence. On this basis, Wang et al (2019a, 2018) considered the size sequence characteristics of the BPA values within each evidence body, and used a sort-factor to modify the credibility degree calculated based on the Jousselme distance function, making the measure of the conflict degree more accurate and reasonable. Therefore, this paper also used the Jousselme distance function and the Sort-Factor to calculate the credibility degree of evidence.

Another factor that cannot be ignored in the assignment of evidence weight is the ambiguity degree (or clarity degree) of evidence. Some scholars use entropy to directly calculate the ambiguity degree of evidence (Rényi, 1961; Deng, 2016, 2020b; Cao et al, 2020; Ni et al, 2020; Luo and Deng, 2020). Deng entropy (Deng, 2016, 2020a; 
Song and Deng, 2021) is representative, which can be used in many fields, such as fuzzy multi-criteria decision-making (Xiao, 2020a). At the same time, some scholars measure the ambiguity degree of evidence from the perspective of generalized information quality (Xiao, 2021b); and other scholars measure the ambiguity degree of evidence from the perspective of divergence (Xiao, 2020b). For example, Xiao (2019) extended the classic JensenShannon (JS) divergence to the belief function, however, the relationship between the focus elements was not considered in the method. Then, Xiao (2020b) proposed a new method of belief function divergence to measure the ambiguity degree of evidence. However, in the process of ambiguity degree calculation, the existing methods generally have such problems as complicated calculation and insufficient use of the information in the body of evidence. Besides, in the process of weight assignment, the researchers usually pay less attention to the ambiguity measurement than conflicting measurement. Therefore, the current weight calculation is mainly based on the conflict degree, without sufficient consideration of the ambiguity degree. In our research, the significant effect on the evidence weight of the ambiguity is firstly verified through test and then a new attempt of the ambiguity measurement and the combination rules based on it is presented. In our scheme, the clarity degree of evidence is computed and used to measure the ambiguity indirectly.

\section{Preliminaries}

\subsection{Basis of D-S evidence theory}

D-S evidence theory is a powerful decision-making tool to reasonably describe unknown information and effectively deal with uncertain information. It mainly selects the values in the interval $[0,1]$ composed of two basic concepts: reliability function and plausibility function as the evidence collected by the decision-maker in the sensors, and uses the Dempster combination rule to fuse the basic probability assignment functions generated by different evidences. D-S evidence theory defines such basic concepts as the identification framework $\Theta$ and Basic probability assignment (BPA) function to describe uncertainty problems. The information combination process of D-S evidence theory is shown in Figure 3.

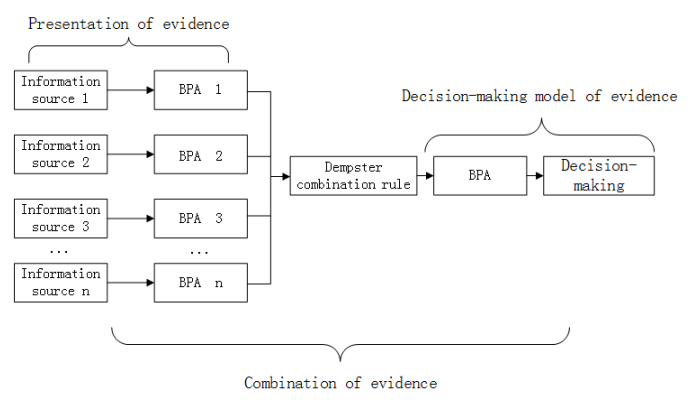

Fig. 3 Basic framework of information combination based on D-S evidence theory

\subsubsection{Frame of discernment}

The D-S evidence theory is built on a finite, nonempty and exclusive set, which is called the 
identification framework and denoted by $\Theta$. The framework consists of $n$ elements that are mutually exclusive. The discernment frame can be expressed as follows:

$$
\Theta=\left\{A_{1}, A_{2}, \cdots, A_{n}\right\}
$$

where $A_{i}$ is called an event or element of the discernment frame $\Theta$.

$$
\begin{aligned}
\Omega= & \left\{\varnothing,\left\{A_{1}\right\},\left\{A_{2}\right\}, \cdots,\left\{A_{n}\right\},\left\{A_{1}, A_{2}\right\},\right. \\
& \left.\left\{A_{1}, A_{3}\right\}, \cdots, \Theta\right\}
\end{aligned}
$$

where $\varnothing$ is an empty set.

\subsubsection{Basic probability assignment}

The basic probability assignment (BPA) function $m$ denotes the mapping from a set $\Omega$ to $[0,1]$. A denotes any subset of the discernment frame $\Theta$, $A \subseteq \Theta$, satisfying the following conditions:

$$
\left\{\begin{aligned}
m(\varnothing) & =0 \\
\sum_{A \subseteq \Theta} m(A) & =1
\end{aligned}\right.
$$

When $m(A)>0, A$ is the focal element of evidence.

\subsubsection{Dempster combination rule}

Assuming that $m_{1}, m_{2}, \cdots, m_{n}$ are the $n$ BPA functions under the same discernment framework $\Theta$, where the focal element is denoted as $A_{i}(i=$
$1,2, \cdots, N)$, then the Dempster combination rule is:

$$
m(A)= \begin{cases}\frac{\sum_{\cap A_{i}=A} \prod_{1 \leq i \leq N} m_{i}\left(A_{i}\right)}{1-K}, & A \neq \varnothing \\ 0 . & A=\varnothing\end{cases}
$$

where the conflict coefficient is $K=$ $\sum_{\cap A_{i}=\varnothing} \prod_{1 \leq i \leq N} m_{i}\left(A_{i}\right)$.

\subsection{Credibility degree and ambiguity degree of evidence}

\subsubsection{Credibility degree of evidence}

The credibility degree of evidence can be described by the evidence similarity calculated by the distance function formula. Wang et al (2017) proposed the three conditions that the evidence similarity function should meet: nonnegativity, disorder and triangulation. Among them, nonnegative represents that the similarity result is always positive; the disordered representative calculation results should be independent of the parameter order; the triangularity represents that the indirect similarity needs to be larger than the direct similarity to satisfy the compactness of the value domain. Based on the above properties, many scholars have proposed some formulas to calculate the similarity between evidence and described the credibility degree of the evidence according to it. The calculation method of the 
credibility degree used in this paper is proposed by Wang et al (2019a), where the initial credibility degree is derived by firstly calculating the similarity of the evidence based on the Jousselme distance function, and then modifying the initial credibility degree based on the Sort-Factor of the evidence., in which the evidence similarity is firstly calculated and defined as follows:

$$
\begin{aligned}
\operatorname{sim}\left(m_{i}, m_{j}\right) & =1-d_{i, j} \\
& =1-\sqrt{\frac{1}{2}\left(m_{i}-m_{j}\right)^{T} D\left(m_{i}-m_{j}\right)}
\end{aligned}
$$

Define the initial credibility of the evidence based on the similarity of the evidence as follows:

$$
\operatorname{Cred}^{\prime}\left(m_{i}\right)=\sum_{m_{j} \in m ; m_{j} \neq m_{i}} \operatorname{sim}\left(m_{j}, m_{i}\right)
$$

where $m_{s}$ is the set of evidence involved in combination. All the evidence in $m_{s}$ must be in the same discrimination framework as $m$. The credibility of the evidence is the overall representation of its similarity. The higher the sum of the similarity of the evidence with others, the higher its credibility is.

The modified credibility degree formula with the Sort-Factor is as follows:
$\operatorname{Cred}\left(m_{i}\right)=\frac{\operatorname{SortFactor}\left(m_{i}\right) * \operatorname{Cred}^{\prime}\left(m_{i}\right)}{\sum_{m_{i} \in m} \operatorname{SortFactor}\left(m_{j}\right) * \operatorname{Cred}^{\prime}\left(m_{j}\right)}$

where SortFactor $\left(m_{i}\right)$ represents the Sort-Factor corresponding to the evidence $m_{i}$.

By using the Jousselme distance and SortFactor to measure the credibility degree of evidence, we can measure the conflict degree of evidence more accurately, considering the correlation between evidence and the amount of information in the evidence body.

\subsubsection{Ambiguity degree of evidence}

The evidence in the evidence body space is often described in ambiguous language, so it is difficult to make a decision directly. We call this kind of ambiguous and fuzzy information as fuzzy evidence. Assuming the frame of discernment $\Theta=\{A, B, C\}, m_{1}: m_{1}(A)=0.1, m_{1}(B)=$ $0.3, m_{1}(C)=0.2, m_{1}(A B)=0.4$. Evidence like $m_{1}$, which does not explicitly state the target category, is a piece of fuzzy evidence. The process of measuring the degree of evidence ambiguity in D-S evidence theory is called Ambiguity Measure (AM) (Deng, 2020b), in which information entropy is used to measure ambiguity of evidence directly. The widely used information entropies are the classical Shannon entropy and Dun entropy, which are calculated as follows:

\section{Shannon entropy}




$$
\operatorname{En}\left(m_{i}\right)=-\sum_{A \in \Omega} m_{i}(A) \log _{2} m_{i}(A)
$$

where $\Omega$ represents the power set space of the frame of discernment, $A$ represents a focus element in the frame of discrimination, $m_{i}(A)$ represents the value of the focus element $A$ corresponding to the evidence body $m_{i}$.

\section{Deng entropy}

$$
E n\left(m_{i}\right)=-\sum_{A \in \Omega} m_{i}(A) \log _{2} \frac{m_{i}(A)}{2^{|A|}-1}
$$

where $|A|$ represents the number of elements in the focus element.

\subsection{Assignment of evidence weight}

The weight of evidence is comprehensively determined by the degree of conflict and ambiguity of evidence. At present, there are two formulas to obtain the weight.

The first one is the direct multiplication of credibility degree and information entropy ( $\mathrm{Li}$ and Xiao, 2020; Wang et al, 2021), which can be expressed as:

$$
w\left(m_{i}\right)=\frac{\operatorname{Cred}\left(m_{i}\right) * \operatorname{En}\left(m_{i}\right)}{\sum_{j} \operatorname{Cred}\left(m_{j}\right) * \operatorname{En}\left(m_{j}\right)}
$$

where $E n\left(m_{i}\right)$ represents the information entropy of the evidence. The fusion formula reflects that the larger the information entropy, the higher the uncertainty is and thus the higher the weight is. This is actually unconventional. We have found the negative correlation between uncertainty and evidence weight through experiments, as detailed in Section 5.1.

The second one is the exponential fusion of credibility degree and information entropy indices (Wang et al, 2019a; Han et al, 2011), which can be expressed as:

$$
w\left(m_{i}\right)=\frac{\operatorname{Cred}\left(m_{i}\right) * \operatorname{En}\left(m_{i}\right)^{-\Delta \operatorname{Cred}\left(m_{i}\right)}}{\sum_{j} \operatorname{Cred}\left(m_{j}\right) * \operatorname{En}\left(m_{j}\right)^{-\Delta \operatorname{Cred}\left(m_{j}\right)}}
$$

$$
\Delta \operatorname{Cred}\left(m_{i}\right)=\operatorname{Cred}\left(m_{i}\right)-\frac{1}{n} \sum_{j=1}^{n} \operatorname{Cred}\left(m_{j}\right)
$$

where $\Delta \operatorname{Cred}\left(m_{i}\right)$ denotes the difference between the credibility degree of evidence $m_{i}$ and the average credibility degree. This method simply takes ambiguity degree of evidence as the modification range of credibility degree, enhancing the weights above the average credibility degree and weakening the weights below it. The larger the ambiguity degree is, the smaller the modification range is. Accordingly, the smaller the ambiguity degree is, the larger the modification range is. However, 
this method ignores the importance of ambiguity measure to decision-making results.

\subsection{Wasserstein Distance}

The idea of Wasserstein distance is derived from optimal transport theory (Villani, 2009). In DataBase systems and Logic Programming (DBLP), it's shown that the study of Wasserstein distance has been blown up since 2017. At present, it exists as an important indicator of the independence of statistical variables in information theory. The Wasserstein distance measures the distance between two variables in terms of probability distribution (Shen et al, 2018). This distance is defined on the metric space $(M, \rho)$, with $\rho(x, y)$ denoting the distance function of two samples $x$ and $y$ in the set $M$. The Wasserstein distance between two continuous distributions $P_{1}(x)$ and $P_{2}(y)$ is defined as follows:

$$
W_{p}\left(P_{1}, P_{2}\right)=\left(\inf _{u \in \Gamma\left(P_{1}, P_{2}\right)} \int \rho(x, y)^{p} d u(x, y)\right)^{\frac{1}{p}}
$$

where $\Gamma\left(P_{1}, P_{2}\right)$ is the set of all joint distributions with $P_{1}(x)$ and $P_{1}(y)$ as marginal distributions within the set $M \times M$, and inf represents the infimum of the expression.

The greatest advantage of the Wasserstein distance is that when two distributions do not intersect or when the intersection is very small, it can still reflect the proximity of the two distributions.
Calculating the distance by Wasserstein distance between evidences in evidence theory can more appropriately reflect the degree of discord between evidences collected in uncertain environment.

When the system discrimination framework is $\Theta=A_{1}, A_{2}, \cdots, A_{n}$, and there are $N$ pieces of evidence $E_{1}, E_{2}, \cdots, E_{N}$ in the system, the corresponding $m$ functions are $m_{1}, m_{2}, \cdots, m_{N}$, where $m_{i}=\left\{m_{i}\left(A_{1}\right), m_{i}\left(A_{2}\right), \cdots, m_{i}\left(A_{n}\right)\right\}$. Assuming that two of the evidences are $E_{i}, E_{j}$, the Wasserstein distance between the evidences $E_{i}, E_{j}$ is:

$$
W\left(E_{i}, E_{j}\right)=\inf _{\gamma \sim \prod\left(E_{i}, E_{j}\right)} \mathbb{E}_{(x, y) \sim \gamma}[\|x-y\|]
$$

where $W\left(E_{i}, E_{j}\right)$ denotes the difference of probability distribution between $E_{i}, E_{j}$, i.e., the distance between the two evidences. And the discrimination frame contains $n$ elements, where the value of $\gamma$ takes a range of $\left(2^{n}-1\right)^{2}$.

\section{Proposed method}

On the basis of effectively measuring the degree of evidence conflict, we studied the influence of the ambiguity degree of evidence on the weight and proposed a new weight assignment method to effectively reduce the effect of fuzzy evidence. The specific architecture of the evidence combination 
based on this weight assignment way is shown in Figure 4.
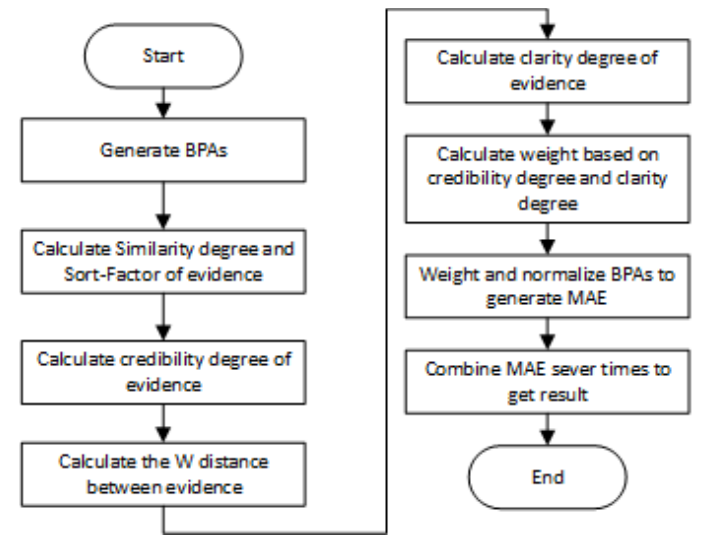

Fig. 4 The flow chart of evidence combination in this paper

Firstly, we obtained the set of BPA using the basic probability assignment generation method based on the number of intervals according to Kang et al (2012). And then compute the credibility degree of evidence using the method in Wang et al (2019a). At the same time, Wasserstein distance is used to calculate the difference between original evidence and uniform distribution to represent the clarity degree of evidence. Then the evidence weights are determined according to the credibility degree and clarity degree. In order to reduce the impact of the conflict evidence and fuzzy evidence on the combination results, the Modified Average Evidence (MAE) is obtained by using the weight to modify the BPA of each evidence. Finally, the decision-making results are obtained by combining MAE $N-1$ times as the Dempster combination formula.
We found the ambiguous evidence has the same important impact on combination results as the conflicting ones, as shown in the following experiment 1 in Section 5. So, in order to improve the effectiveness of the evidence modification, our weight assignment formula makes the gain and penalty to the evidence weights of both the credibility degree and clarity degree keep the same trend.

\subsection{Clarity degree of evidence calculation based on Wasserstein distance}

To obtain the clarity degree of evidence, we calculated the distance between evidence and the uniform distribution as the Wasserstein distance formula 14 at first, and then normalized the results. The clarity degree calculation formula of evidence $m_{i}$ in our scheme is as follows:

$$
\begin{gathered}
\operatorname{Clar}\left(m_{i}\right)=W\left(m_{i}, U\right) \\
U=\left(\frac{1}{|m|}, \frac{1}{|m|}, \cdots, \frac{1}{|m|}\right), U \in \mathbb{R}^{|m|}
\end{gathered}
$$

where $m_{i}$ represents the evidence to be calculated; $U$ represents uniform distribution; $|m|$ represents the number of focus elements in any evidence body; and $C l a r\left(m_{i}\right)$ represents the clarity degree of the evidence $m_{i}$. 


\subsection{Weight assignment based on credibility and clarity degree of evidence}

Based on the credibility degree and clarity degree of evidence, we propose a new weight assignment formula:

$$
w\left(m_{i}\right)=\operatorname{Cred}\left(m_{i}\right) * \frac{N * \operatorname{Clar}\left(m_{i}\right)}{\sum_{j} \operatorname{Clar}\left(m_{j}\right)}
$$

where $N$ represents the number of evidence involved in the combination and $w\left(m_{i}\right)$ represents the weight of evidence $m_{i}$.

\subsection{Combination rules}

Based on the weight of evidence, the modified average evidence (MAE) is the evidence corrected according to the weight. Normalized evidence MAE is defined as follows:

Assuming that $M_{1}, m_{2}, \cdots, m_{N}$ is $N$ evidences in the same discernment framework, and $w\left(m_{i}\right)$ is the weight of evidence $m_{i}$, then the evidence MAE is:

$$
M A E=\sum_{m_{i} \in m} m_{i} \times w\left(m_{i}\right)
$$

The final combination result can be obtained by fusing MAE $n-1$ times using the basic formula. Its process is shown in Figure 5.

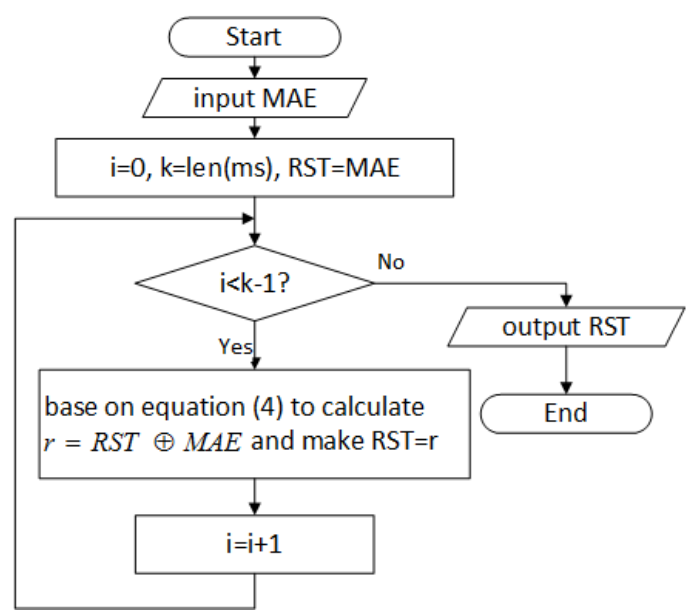

Fig. 5 MAE to combination results process

\section{Experiment}

In the experimental section, we designed three groups of experiments. Experiment 1 verified the conclusion mentioned above: fuzzy evidence does have an important influence on the final decisionmaking. Experiments 2 and 3 respectively verified the effectiveness and superiority of the proposed method by comparing with other methods.

\subsection{Experiment 1}

It is assumed that $m_{1}, m_{2}, m_{3}, m_{4}$ are four pieces of evidence in the same frame of discernment, and the contents of each evidence body are shown in Table 1. It can be seen that the results of the four pieces of evidences all point to A. Now we make a fifth piece of evidence to combine with the above four and compute the credibility degree, clarity degree for all the evidence and the BPAs of focal elements after the combination as 
well. In the fifth piece of evidence, each focal element can take value every 0.02 between 0 and 1 . Then, there can be 23426 possibilities for the fifth evidence. In order to test the importance of ambiguity measurement, we only used the credibility degree to determine the evidence weight in this experiment and observed the relationship between the combination results and the properties of evidence bodies. The complete combination process is shown in Algorithm 1 for detail. Thus, A scatterplot of the corresponding relationship between the BPA value of target $A$ and the credibility degree and clarity degree of the fifth piece of evidence is shown in Figure 6.

Table 1 The BPA value of the focal element in each evidence body.

\begin{tabular}{lcccc}
\hline Focus Element & $m(A)$ & $m(B)$ & $m(C)$ & $m(\Theta)$ \\
\hline$m_{1}$ & 0.70 & 0.10 & 0 & 0.20 \\
$m_{2}$ & 0.70 & 0 & 0 & 0.30 \\
$m_{3}$ & 0.65 & 0.15 & 0 & 0.20 \\
$m_{4}$ & 0.75 & 0.05 & 0 & 0.20 \\
\hline
\end{tabular}

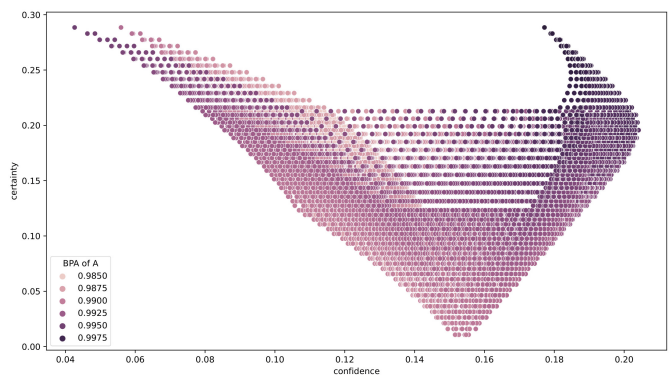

Fig. 6 Scatter plot of BPA values of target A

The darker color of the dots in Figure 6 means that the BPA value is larger, and the horizontal

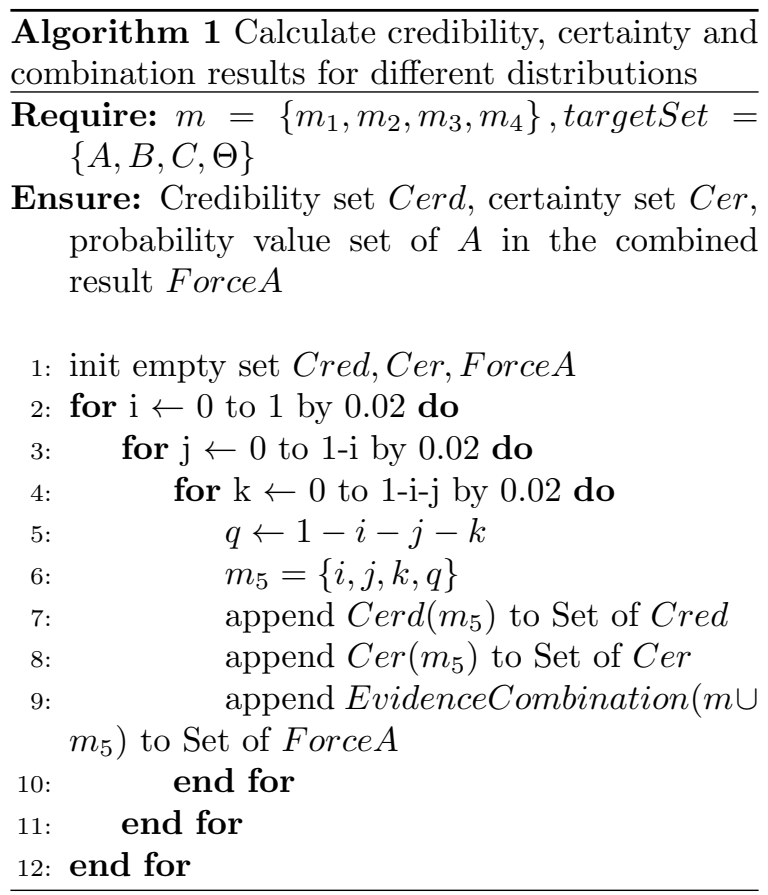

coordinate represents the credibility degree of evidence, while the vertical coordinate represents the clarity degree of the evidence obtained based on the $\mathrm{W}$ distance formula. It can be seen from the scatterplot that the BPA value of the dots with high credibility degree and low clarity degree is smaller than that of the ones with low credibility degree and high clarity degree. This phenomenon indicates that the ambiguity measurement of evidence absolutely has an important influence on the final decision, and this influence is not less than the conflict measurement of evidence has. Therefore, the influence of fuzzy evidence should be taken into more account in the weight distribution.

When $m_{a}, m_{b}$ and $m_{c}$ in Table 2 are used as the fifth piece of evidence respectively, the combination results are shown in Table 3. 
Table 2 BPA values for each focal element in the three different the fifth piece of evidence.

\begin{tabular}{lcccc}
\hline Focus Element & $m(A)$ & $m(B)$ & $m(C)$ & $m(\Theta)$ \\
\hline$m_{a}$ & 0.80 & 0.20 & 0 & 0 \\
$m_{b}$ & 0 & 0.20 & 0.80 & 0 \\
$m_{c}$ & 0.25 & 0.25 & 0.25 & 0.25 \\
\hline
\end{tabular}

Table 3 Combined results after adding the fifth piece of evidence.

\begin{tabular}{lcccc}
\hline Combined Focus Elements & $m(A)$ & $m(B)$ & $m(C)$ & $m(\Theta)$ \\
\hline$m_{1} \oplus m_{2} \oplus m_{3} \oplus m_{4} \oplus m_{a}$ & 0.997 & 0.003 & 0 & 0 \\
$m_{1} \oplus m_{2} \oplus m_{3} \oplus m_{4} \oplus m_{b}$ & 0.993 & 0.004 & 0.002 & 0.001 \\
$m_{1} \oplus m_{2} \oplus m_{3} \oplus m_{4} \oplus m_{c}$ & 0.990 & 0.007 & 0.002 & 0.001
\end{tabular}

It can be seen from the table that the value of $M(A)$ decreases when the combined evidence contains conflicting evidence or fuzzy evidence, i. e., both conflict evidence and fuzzy evidence have influence on the final decision. However, when there is fuzzy evidence in the evidence to be combined, the value of $M(A)$ decreases larger. Therefore, we can also draw the conclusion as above that the fuzzy evidence has great a impact on the final decision and should be fully considered when determining the weight of evidence.

\subsection{Experiment 2}

In this experiment, we take the automobile system fault as an example to predict the fault type, and illustrate the effectiveness and feasibility of the proposed method. The data used in the experiment adds a piece of fuzzy evidence $m_{6}$ on the basis of that Li and Xiao (2020) used. It contains 6 pieces of evidence and 3 fault types. Assuming the discernment frame $\Theta=\{A, B, C\}$, the fault diagnosis results are shown in Table 4, The correct fault type is A. We respectively used the methods in reference Wang et al (2019a); Li and Xiao (2020); Wang et al (2021), and the method we have proposed to combine this evidence and got the BPA values of each focus element as listed in Table 5.

Table 4 The BPA value of the focal element in each evidence body.

\begin{tabular}{lcccc}
\hline Focus Element & $m(A)$ & $m(B)$ & $m(C)$ & $m(\Theta)$ \\
\hline$m_{1}$ & 0.70 & 0.10 & 0 & 0.20 \\
$m_{2}$ & 0.70 & 0 & 0 & 0.30 \\
$m_{3}$ & 0.65 & 0.15 & 0 & 0.20 \\
$m_{4}$ & 0.75 & 0.05 & 0 & 0.20 \\
$m_{5}$ & 0 & 0.20 & 0.80 & 0 \\
$m_{6}$ & 0.3 & 0.23 & 0.22 & 0.25 \\
\hline
\end{tabular}

As shown in Table 4, the diagnosis of $m_{5}$ conflicts with other evidence, that is, it's conflicting evidence. While the diagnosis of $m_{6}$ is fuzzy evidence which is difficult for a judgment because it does not explicitly point to any fault type. The combined results may be distorted by such highly uncertain evidence. In Table 5, there is the weight of evidence calculated by reference Wang et al (2019a); Li and Xiao (2020); Wang et al (2021) and the method in this paper. Table 6 shows the results of different evidence combination methods after each fusion.

As shown in Table 6, when there is uncertain evidence, the classical Dempster combination rule is greatly affected and cannot make an accurate 
Table 5 Each weight of evidence assigned by the different methods.

\begin{tabular}{lcccccc}
\hline Method & $m_{1}$ & $m_{2}$ & $m_{3}$ & $m_{4}$ & $m_{5}$ & $m_{6}$ \\
\hline Wang et al (2019a) & 0.335 & 0.127 & 0.329 & 0.128 & 0.007 & 0.075 \\
Li and Xiao (2020) & 0.157 & 0.155 & 0.176 & 0.130 & 0.032 & 0.351 \\
Wang et al (2021) & 0.180 & 0.144 & 0.202 & 0.145 & 0.019 & 0.311 \\
Proposed method & 0.326 & 0.180 & 0.288 & 0.181 & 0.015 & 0.011 \\
\hline
\end{tabular}

Table 6 The BPA value of each focal element after evidence combination by different methods.

\begin{tabular}{lcccccc}
\hline \multirow{2}{*}{ Method } & & & & & \\
& Targets & $m_{1} \oplus m_{2}$ & $\begin{array}{c}m \uparrow m_{2} \\
\oplus m_{3}\end{array}$ & $\begin{array}{c}m_{1} \oplus m_{2} \oplus \\
m_{3} \oplus m_{5}\end{array}$ & $\begin{array}{c}m_{1} \oplus m_{2} \\
\oplus m_{3} \oplus m_{5} \\
\oplus m_{6}\end{array}$ & $\begin{array}{c}m_{1} \oplus m_{3} \oplus m_{5} \\
\oplus m_{6} \oplus m_{4}\end{array}$ \\
& & & & & 0.0000 & 0.0000 \\
Dempster & $\mathrm{A}$ & 0.9032 & 0.9598 & 0.0000 & 0.4013 & 0.4559 \\
& $\mathrm{~B}$ & 0.0323 & 0.0249 & 0.3962 & 0.5987 & 0.5441 \\
& $\mathrm{C}$ & 0.0000 & 0.0000 & 0.6038 & 0.0000 & 0.0000 \\
Wang et al (2019a) & $\Theta$ & 0.0645 & 0.0153 & 0.0000 & 0.9905 & 0.9972 \\
& $\mathrm{~A}$ & 0.8998 & 0.9554 & 0.9830 & 0.0077 & 0.0024 \\
& $\mathrm{~B}$ & 0.0426 & 0.0310 & 0.0133 & 0.0007 & 0.0002 \\
Li and Xiao (2020) & $\mathrm{C}$ & 0.0000 & 0.0000 & 0.0004 & 0.0010 & 0.0002 \\
& $\Theta$ & 0.0576 & 0.0136 & 0.0033 & 0.9450 & 0.9828 \\
& $\mathrm{~A}$ & 0.9032 & 0.9589 & 0.9779 & 0.0320 & 0.0107 \\
& $\mathrm{~B}$ & 0.0298 & 0.0252 & 0.0129 & 0.0029 & 0.0007 \\
Wang et al (2021) & $\mathrm{C}$ & 0.0000 & 0.0000 & 0.0051 & 0.9593 & 0.9882 \\
& $\Theta$ & 0.0670 & 0.0159 & 0.0041 & 0.0260 & 0.0081 \\
& $\mathrm{~A}$ & 0.6138 & 0.7015 & 0.8848 & 0.0121 & 0.0031 \\
& $\mathrm{~B}$ & 0.1780 & 0.1466 & 0.0574 & 0.0025 & 0.0006 \\
& $\mathrm{C}$ & 0.1271 & 0.1228 & 0.0473 & $\mathbf{0 . 9 9 3 5}$ & $\mathbf{0 . 9 9 8 3}$ \\
& $\Theta$ & 0.0811 & 0.0291 & 0.0105 & 0.0014 \\
& $\mathrm{~A}$ & $\mathbf{0 . 9 0 1 1}$ & $\mathbf{0 . 9 5 7 7}$ & $\mathbf{0 . 9 8 3 0}$ & 0.0051 & 0.0001 \\
& $\mathrm{~B}$ & 0.0379 & 0.0278 & 0.0122 & 0.0004 & 0.0002 \\
\hline
\end{tabular}

judgment, while other methods can still successfully identify the correct fault type. From Table 5 and Table 6 , we can see that all the methods except Dempster can accurately identify the conflicting evidence $m_{5}$ and assign it smaller weights. However, for the fuzzy evidence $m_{6}$, the method of Li and Xiao (2020) and Wang et al (2021) cannot effectively deal with it in their weight assignment method, which resulted in a decrease in the BPA value of focus element $\mathrm{A}$ after evidence being combined with $m_{6}$. It's obvious that the fuzzy evidence m6 did not affect the combination result in Wang et al (2019a) and our method because these two methods assigned a very small weight to it. In comparison, the weight of fuzzy evidence assigned by our method is smaller, and the BPA value of 
focus element A is higher as well. To be concluded, the proposed method can assign more reasonable weights to the evidence than all the other methods when both conflicting evidence and fuzzy evidence exist.

Besides, the proposed method has better convergence because the BPA value of the target focus element is always higher in our method than in other methods after each round combination.

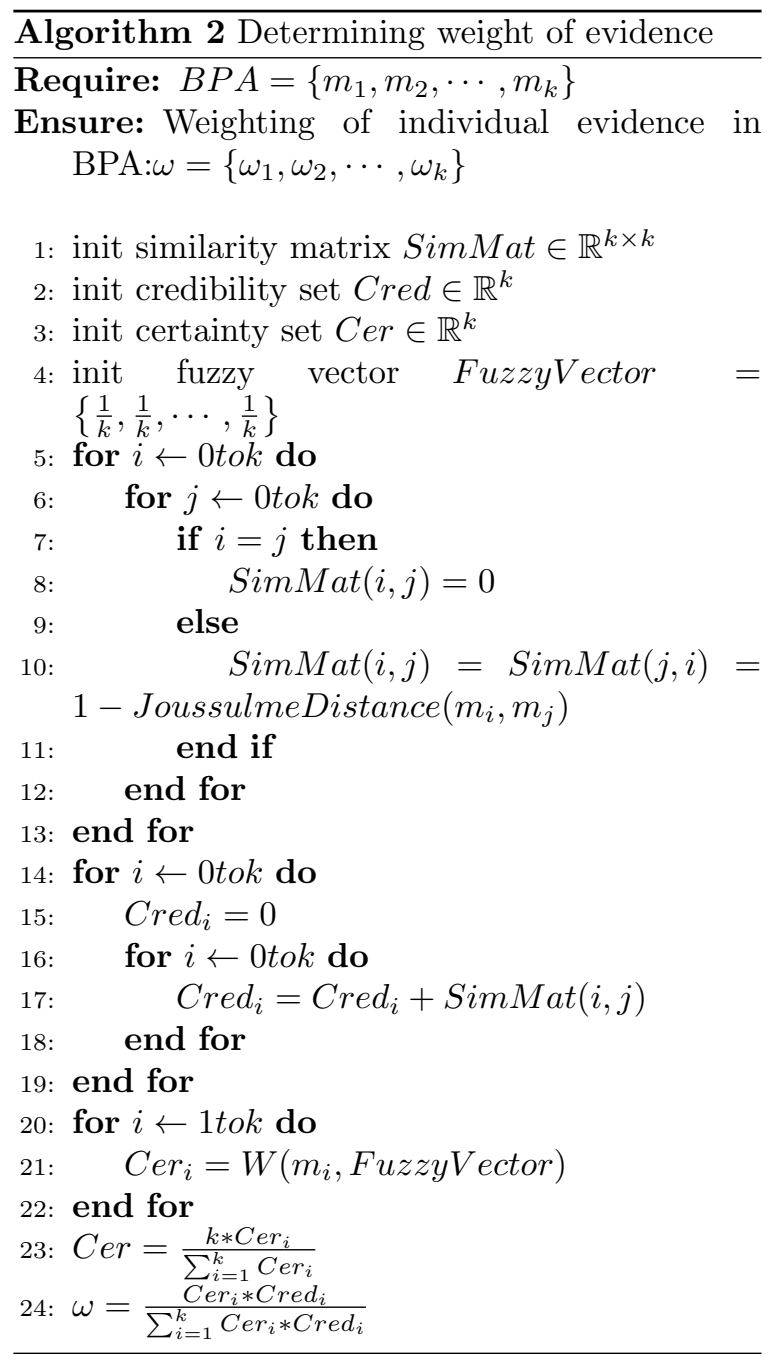

\subsection{Experiment 3}

In this experiment, we compare the decision accuracy and F1 score of the classical Dempster Combination rule, the method in reference Wang et al (2019a); Li and Xiao (2020); Wang et al (2021) and our proposed method. The Iris data set commonly used in this field was used in the experiment. There are 150 data pieces in this data set, in which each piece of data contains five values: namely sepal length (SL), sepal width $(\mathrm{SW})$, petal length $(\mathrm{PL})$, petal width $(\mathrm{PW})$ and plant type. There are three kinds of plant types: Setosa (Se), Versicolor (Ve) and Virginica (Vi). The first four attribute values can be transformed into four pieces of evidences, and the discernment frame is $\Theta=\{S e, V e, V i\}$, then the plant type can be predicted by using different combination rules. The performance of these methods can be compared with the accuracy and F1 score of decision-making.

Firstly, generate evidence. Four attribute values in the Iris dataset were converted into four pieces of evidence using the BPA generation method proposed by Kang et al (2012). We randomly selected a piece of data, where the values of SL, SW, PL and PW were 5.0, 3.5, 1.3 and 0.3 respectively, and the plant type was Setosa. The values of the focal elements in the body of evidence transformed into evidence are shown in Table 7. 
Table 7 BPA values for each focal element in the three different the fifth piece of evidence.

\begin{tabular}{lcccc}
\hline focus element & SL & SW & PL & PW \\
\hline Se & 0.2200 & 0.1642 & 0.7171 & 0.6343 \\
$\mathrm{Ve}$ & 0.0981 & 0.1163 & 0.1122 & 0.1438 \\
$\mathrm{Vi}$ & 0.0642 & 0.1538 & 0.0758 & 0.0990 \\
$\mathrm{Ve}, \mathrm{Vi}$ & 0.0852 & 0.1289 & 0.0943 & 0.1226 \\
$\mathrm{Se}, \mathrm{Vi}$ & 0.1563 & 0.1642 & 0.0002 & 0.0001 \\
$\mathrm{Se}, \mathrm{Ve}$ & 0.2200 & 0.1363 & 0.0002 & 0.0001 \\
$\mathrm{Se}, \mathrm{Ve}, \mathrm{Vi}$ & 0.1563 & 0.1363 & 0.0002 & 0.0001 \\
\hline
\end{tabular}

Then, determine the weight of evidence.

According to the data in Table 7, the credibility degree and clarity degree the evidence are calculated, and the weight of evidence is determined. The details of algorithm are shown in Algorithm 2.

\section{Finally, make decisions by using com-}

bination rules. We present a flowchart of the experiment from input data to decision-making, as shown in Figure 7. The decision results for each method are shown in Table 8. And we also counted the change curves of accuracy and F1 score for each method when the size of the training set is changing, as shown in Figure 8.

Figure 8 shows the average results after 100 random samples of the training set. It can be seen from Figure 8 that our proposed method has the highest accuracy, F1 score, and has a significant improvement when compared to other methods.

In order to further verify the effectiveness of our proposed evidence clarity degree calculation method and the rationality of the weight assignment formula based on it, four sets of comparison

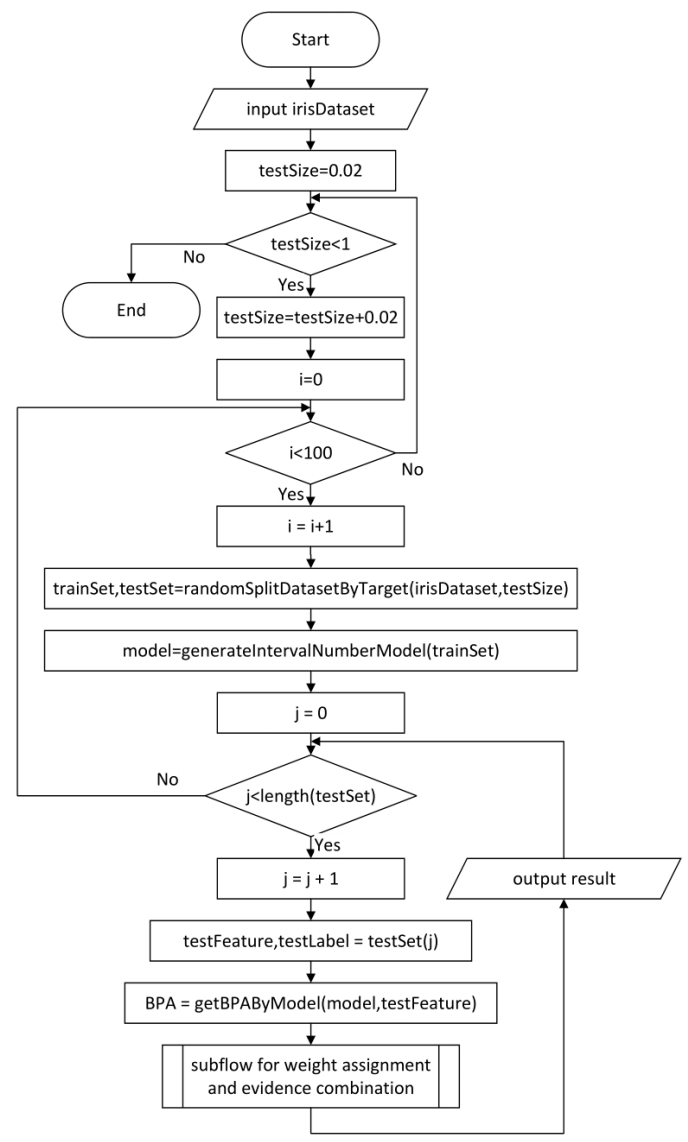

Fig. 7 Comparison of experimental results of different methods

tests were done to modify the evidence using multiple fusions and then observe the accuracy and F1 score of the decision after each fusion. In detail, we fused the clarity degree with the credibility degree for $0,1,2$ and 3 times respectively to determine the weight for modifying evidence. The four decision result curves of different colors shown in the bellowing figures correspond to None, Once, Twice and Triple respectively. It can be seen from the Figure 9 that the accuracy and F1 score of the results will be significantly improved with the increase of clarity fusion times. This is because in 
Table 8 The BPA values of each focal element after evidence combination of different methods

\begin{tabular}{lccccccc}
\hline methods & Se & Ve & Vi & Ve,Vi & Se,Vi & Se,Ve & $\Theta$ \\
\hline Dempster & 0.8900 & 0.0659 & 0.0408 & 0.0032 & 0.0000 & 0.0000 & 0.0000 \\
Wang et al (2019a) & 0.9664 & 0.0212 & 0.0114 & 0.0010 & 0.0000 & 0.0000 & 0.0000 \\
Li and Xiao (2020) & 0.6720 & 0.1594 & 0.1310 & 0.0082 & 0.0125 & 0.0162 & 0.0007 \\
Wang et al (2021) & 0.7591 & 0.1222 & 0.0985 & 0.0064 & 0.0060 & 0.0073 & 0.0004 \\
Proposed method & 0.9802 & 0.0132 & 0.0059 & 0.0006 & 0.0000 & 0.0000 & 0.0000 \\
\hline
\end{tabular}

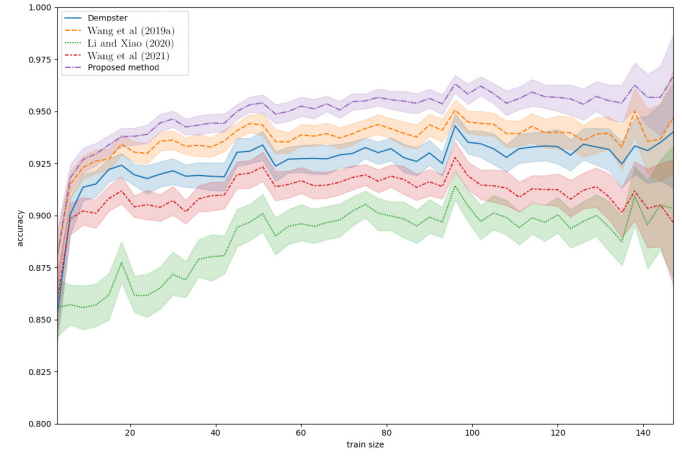

(a) Accuracy Comparison

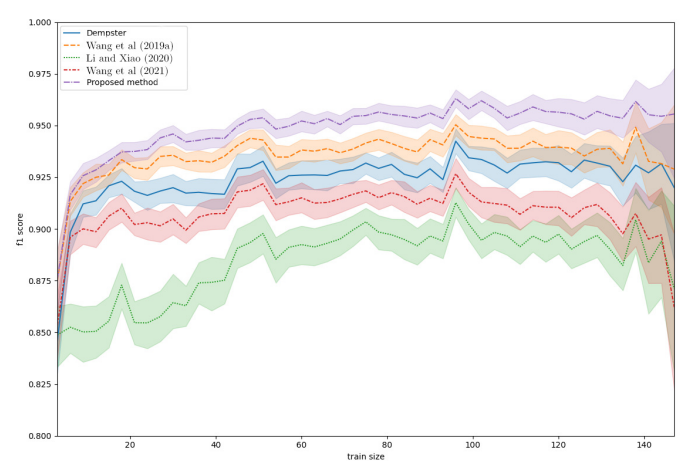

(b) F1 Score Comparison

Fig. 8 Comparison of experimental results of different methods

weight computing, the clarity can play a bigger role in the weight assignment with the increase of its fusion times, which makes the weight of fuzzy evidence smaller.

In general, our weight assignment method can effectively deal with the case where both conflicting and ambiguous evidence exist. Especially, it's

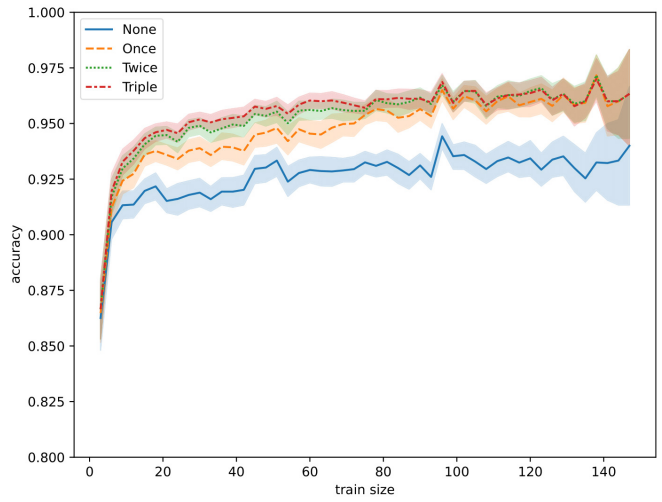

(a) Accuracy Comparison

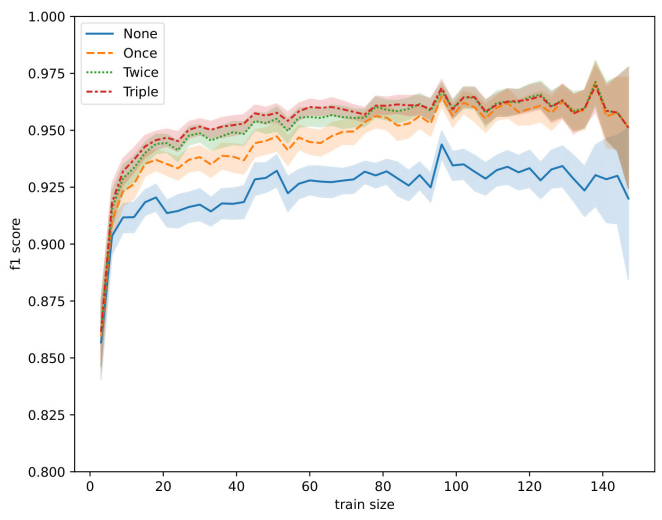

(b) F1 Score Comparison

Fig. 9 Results after multiple fusions of clarity

found to have better performance in real datasets like Iris, which is because the probability of highly fuzzy evidence appearing in the real data set is much greater than that of conflicting evidence. Therefore, the proposed method is superior to 
other approaches on data sets in practice for being good at dealing with fuzzy evidence.

\section{Conclusion}

In this paper, we conducted further research on how to reduce the impact of the conflicting evidence and fuzzy evidence on the combination results in the process of evidence combination. Our main contributions are as follows: (1) We verified that the ambiguity of evidence has a significant impact on the final decision result of evidence combination through experiment; then analyzed and drew the conclusion that the two commonly used weight assignment methods in the current evidence combination rules cannot effectively reduce the influence of ambiguous evidence on the combination result. (2) We introduced the Wasserstein distance into the ambiguity measurement to calculate clarity degree of evidence and proposed a new evidence weight assignment method based on it. By using data from $\mathrm{Li}$ and Xiao (2020) and real Iris data sets, experiments showed that the convergence of the proposed method can reach the best level of other similar methods, while the accuracy of decision-making is much higher than that of other compared methods.

However, since the number of elements in the focus element is not considered when using
Wasserstein distance, there is very little ambiguous evidence that cannot be measured accurately. In future work, we will consider how to make better use of Wasserstein distance to measure the ambiguous evidence more accurately. In addition, we also found in the experiments that multiple uses of clarity degree to fuse with credibility degree can achieve better experimental results; therefore, it is necessary for our further study to seek for an improved fusion formula which is more suitable for the clarity degree and credibility degree.

Author Contribution. WYC was involved in writing - original, visualization, data curation, resources, formal analysis, methodology, and software. WJ contributed to writing-review and supervision. HMJ was involved in software, validation, formal analysis, and data curation. WMH was involved in conceptualization and visualization.

Funding. The work was supported by National Natural Science Foundation of China Grant No. 61972133.

Data availability and material. The data used to support the findings of this study are available from the corresponding author upon request. 


\section{Declarations}

Conflict of Interest. The authors declare that they have no conflict of interest.

Code availability. The implementation of the algorithm in the manuscript will be sent upon request.

Ethical approval. This article does not contain any studies with human participants or animals performed by any of the authors.

\section{References}

Cao ZH, Ding WP, Wang YK, et al (2020) Effects of repetitive SSVEPs on EEG complexity using multiscale inherent fuzzy entropy. Neurocomputing 389:198-206. https://doi.org/10.1016/j.neucom.2018.08.091, URL https://www.sciencedirect.com/science/ article/pii/S0925231219304138

Chen L, Deng Y, Cheong KH (2021) Probability transformation of mass function: A weighted network method based on the ordered visibility graph. Engineering Applications of Artificial Intelligence 105:104,438. https: //doi.org/10.1016/j.engappai.2021.104438, URL https://linkinghub.elsevier.com/retrieve/ pii/S0952197621002864
Dempster AP (1967) Upper and Lower Probabilities Induced by a Multivalued Mapping. The Annals of Mathematical Statistics 38(2):325-339. https://doi.org/10.1214/ aoms/1177698950, URL http://projecteuclid. org/euclid.aoms/1177698950

Deng Y (2016) Deng entropy. Chaos, Solitons \& Fractals 91:549-553. https: //doi.org/10.1016/j.chaos.2016.07.014, URL https://www.sciencedirect.com/science/ article/pii/S0960077916302363

Deng Y (2020a) Information Volume of Mass Function. INTERNATIONAL JOURNAL OF COMPUTERS COMMUNICATIONS \& CONTROL 15(6). https://doi.org/10.15837/ijccc. 2020.6.3983, URL http://univagora.ro/jour/ index.php/ijccc/article/view/3983

Deng Y (2020b) Uncertainty measure in evidence theory. Sci China Inf Sci 63(11):210,201. https: //doi.org/10.1007/s11432-020-3006-9, URL https://doi.org/10.1007/s11432-020-3006-9

Deng Y, Shi Wk (2003) A Modified Combination Rule of Evidence Theory. JOURNAL OF SHANGHAI JIAOTONG UNIVERSITY 37(8):1275-1278. https://doi.org/ 10.3321/j.issn:1006-2467.2003.08.032 
Dubois D, Prade H (1985) Evidence measures based on fuzzy information. Automatica 21(5):547-562. https: //doi.org/10.1016/0005-1098(85)90004-4,

URL https://www.sciencedirect.com/science/ article/pii/0005109885900044

Fei L, Xia J, Feng Y, et al (2019) An ELECTREBased Multiple Criteria Decision Making Method for Supplier Selection Using DempsterShafer Theory. IEEE Access 7:84,701-84,716. https://doi.org/10.1109/ACCESS.2019. 2924945

Fu B, Fang JW, Zhao XL, et al (2021) A Belief Coulomb Force in D-S Evidence Theory. IEEE Access 9:82,979-82,988. https://doi.org/ 10.1109/ACCESS.2021.3086232

Gruyer D, Demmel S, Magnier V, et al (2016) Multi-Hypotheses Tracking using the Dempster-Shafer Theory, application to ambiguous road context. Information Fusion 29:40-56. https://doi.org/10.1016/j.inffus.2015.10.001, URL https://www.sciencedirect.com/science/ article/pii/S1566253515000962

Han DQ, Deng Y, Han CZ, et al (2011) Weighted evidence combination based on distance of evidence and uncertainty measure. Journal of Infrared and Millimeter Waves 30(5):396400,468
Hu Hl, Zhong Qx, Liu L (2016) Improved method to D-S evidence theory based on iterative synthesis. Application Research of Computers 33(10):2985-2987,3044. https://doi.org/10. 3969/j.issn.1001-3695.2016.10.026

Huang Dr, Chai Yc, Zhao L, et al (2018) Traffic Congestion Status Identification Method for Road Network with Multi-source Uncertain Information. Acta Automatica Sinica 44(3):533-544. https://doi.org/10.16383/j.aas. 2018.c160373

Jousselme AL, Grenier D, Bossé É (2001) A new distance between two bodies of evidence. Information Fusion 2(2):91-101. https: //doi.org/10.1016/S1566-2535(01)00026-4, URL https://www.sciencedirect.com/science/ article/pii/S1566253501000264

Kang By, Li Y, Deng Y, et al (2012) Determination of Basic Probability Assignment Based on Interval Numbers and its Application. Acta Electronica Sinica 40(6):1092-1096. https:// doi.org/10.3969/j.issn.0372-2112.2012.06.004

Lefèvre E, Elouedi Z (2013) How to preserve the conflict as an alarm in the combination of belief functions? Decision Support Systems 56:326-333. https://doi.org/10.1016/j.dss.2013. 06.012, URL https://www.sciencedirect.com/ science/article/pii/S0167923613001838 
Lei H, Sheng L (2021) An Improved Algorithm of D-S Evidence Fusion. J Phys: Conf Ser 1871(1):012,108. https://doi.org/10.1088/ 1742-6596/1871/1/012108, URL https:/doi. org/10.1088/1742-6596/1871/1/012108

Li H, Xiao F (2020) A method for combining conflicting evidences with improved distance function and Tsallis entropy. International Journal of Intelligent Systems 35(11):18141830. https://doi.org/10.1002/int.22273, URL https://onlinelibrary.wiley.com/ doi/abs/10.1002/int.22273, _eprint: https://onlinelibrary.wiley.com/doi/pdf/10.1002/int.22273

Li J, Xie B, Jin Y, et al (2020) Weighted Conflict Evidence Combination Method Based on Hellinger Distance and the Belief Entropy. IEEE Access 8:225,507-225,521. https://doi. org/10.1109/ACCESS.2020.3044605

Li Y, Guo Yj, Yang Yl (2014) Identification and application of the evidence conflict based on K-L information distance. Systems Engineering — Theory \& Practice 34(8):2071-2077

Lian C, Ruan S, Denœux T, et al (2019) Joint Tumor Segmentation in PET-CT Images Using Co-Clustering and Fusion Based on Belief Functions. IEEE Transactions on Image Processing 28(2):755-766. https://doi.org/10.1109/ TIP.2018.2872908
Shafer theory and uninorm-based framework

Lin Y, Wang C, Ma C, et al (2016) A new combination method for multisensor conflict information. J Supercomput 72(7):2874-2890. https://doi.org/10.1007/ s11227-016-1681-3, URL https://doi.org/10. 1007/s11227-016-1681-3

Luo Z, Deng Y (2020) A Matrix Method of Basic Belief Assignment's Negation in Dempster-Shafer Theory. IEEE Transactions on Fuzzy Systems 28(9):2270-2276. https://doi. org/10.1109/TFUZZ.2019.2930027 of reasoning and multiattribute decisionmaking for surveillance system. International Journal of Intelligent Systems 34(11):30773104. https://doi.org/10.1002/int.22175, URL https://onlinelibrary.wiley.com/doi/abs/10. 1002/int.22175

Murphy CK (2000) Combining belief functions when evidence conflicts. Decision Support Systems 29(1):1-9. https: //doi.org/10.1016/S0167-9236(99)00084-6, URL https://www.sciencedirect.com/science/ article/pii/S0167923699000846

Ni S, Lei Y, Tang Y (2020) Improved Base Belief Function-Based Conflict Data Fusion Approach Considering Belief Entropy in the Evidence 
Theory. Entropy 22(8):801. https://doi.org/10. 3390/e22080801, URL https://www.mdpi.com/ $1099-4300 / 22 / 8 / 801$

Rényi A (1961) On measures of entropy and information. In: Proceedings of the Fourth Berkeley Symposium on Mathematical Statistics and Probability, Volume 1: Contributions to the Theory of Statistics. University of California Press, pp 547-561

Shafer G (1976) A Mathematical Theory of Evidence. Princeton University Press, https://doi.org/10.1515/9780691214696, URL https://www.degruyter.com/document/doi/10. 1515/9780691214696/html

Shen J, Qu Y, Zhang W, et al (2018) Wasserstein Distance Guided Representation Learning for Domain Adaptation. In: ThirtySecond AAAI Conference on Artificial Intelligence, URL https://www.aaai.org/ocs/index. php/AAAI/AAAI18/paper/view/17155

Smets P (1990) The combination of evidence in the transferable belief model. IEEE Transactions on Pattern Analysis and Machine Intelligence 12(5):447-458. https://doi.org/10.1109/ 34.55104

Song Y, Deng Y (2021) Entropic Explanation of Power Set. INTERNATIONAL JOURNAL OF
COMPUTERS COMMUNICATIONS \& CON-

TROL 16(4). https://doi.org/10.15837/ijccc. 2021.4.4413, URL http://univagora.ro/jour/ index.php/ijccc/article/view/4413

Song Yf, Wang Xd, Lei L, et al (2014) Measurement of evidence conflict based on correlation coefficient. Journal on Communications 35(5):95-100. https://doi.org/10.3969/j. issn.1000-436x.2014.05.013

Sun L, Chang Y, Pu J, et al (2020) A Weighted Evidence Combination Method Based on the Pignistic Probability Distance and Deng Entropy. J Aerosp Technol Manag 12. https://doi.org/10.5028/jatm.v12.1173, URL http://www.scielo.br/j/jatm/a/ LrYktWDVHLcLTsqRwDW5cVy/?lang=en\& format $=\mathrm{html}$

Villani C (2009) Optimal transport: old and new. No. 338 in Grundlehren der mathematischen Wissenschaften, Springer, Berlin, oCLC: ocn244421231

Wang J, Qiao K, Zhang Z, et al (2017) A new conflict management method in Dempster-Shafer theory. International Journal of Distributed Sensor Networks 13(3):1550147717696,506. https: //doi.org/10.1177/1550147717696506, URL https://doi.org/10.1177/1550147717696506 
Wang J, Zhang Zy, Qiao Ky (2018) Evidence combination rule with similarity collision reduced. Journal of Computer Applications 38(10):2794-2800. https://doi.org/10.11772/ j.issn.1001-9081.2018030532, URL https:// kns.cnki.net $/ \mathrm{kcms} /$ detail/detail.aspx?dbcode $=$ CJFD\&dbname $=$ CJFDLAST2018\&filename $=$ JSJY201810009\&uniplatform $=$ NZKPT\&v $=$ aKeECELjBbJtIEsXAWnzB4hw1fPCdHCju78L5p8fovPdQQk2yE4NoCk\%

25mmd2BhDMUpslD

Wang J, Qiao K, Zhang Z (2019a) An improvement for combination rule in evidence theory. Future Generation Computer Systems 91:1-9. https://doi.org/10.1016/j.future.2018. 08.010, URL https://www.sciencedirect.com/ science/article/pii/S0167739X18300839

Wang X, Di P, Yin Dl (2021) Conflict Evidence Fusion Method Based on Lance Distance and Credibility Entropy. Systems Engineering and Electronics pp 1-14

Wang Y, Liu F, Zhu A (2019b) Bearing Fault Diagnosis Based on a Hybrid Classifier Ensemble Approach and the Improved DempsterShafer Theory. Sensors 19(9):2097. https:// doi.org/10.3390/s19092097, URL https://www. mdpi.com/1424-8220/19/9/2097

Wang Z, Hu Wd, Yu Wx, et al (2001) A Combination Rule of Conflict Evidence Based on
Proportional Belief Assignment. ACTA ELECTRONICA SINICA 29(z1):1852-1855. https:// doi.org/10.3321/j.issn:0372-2112.2001.z1.030

Wei Yc (2011) An Improved D-S Evidence Combination Method Based on K-L Distance. TELECOMMUNICATION ENGINEERING 51(1):27-30. https: //doi.org/10.3969/j.issn.1001-893x.2011.01.006 Xiao F (2019) Multi-sensor data fusion based on the belief divergence measure of evidences and the belief entropy. Information Fusion 46:23-32. https://doi.org/10.1016/j.inffus.2018. 04.003, URL https://www.sciencedirect.com/ science/article/pii/S1566253517305584

\section{Xiao F (2020a) EFMCDM: Evidential Fuzzy Multicriteria Decision Making Based on Belief Entropy. IEEE Transac- tions on Fuzzy Systems 28(7):1477-1491. https://doi.org/10.1109/TFUZZ.2019.2936368}

Xiao F (2020b) A new divergence measure for belief functions in D-S evidence theory for multisensor data fusion. Information Sciences 514:462-483. https://doi.org/10.1016/j.ins. 2019.11.022, URL https://www.sciencedirect. com/science/article/pii/S0020025519310679

Xiao F (2021a) CEQD: A Complex Mass Function to Predict Interference Effects. IEEE Transactions on Cybernetics pp 1-13. https://doi. 
org/10.1109/TCYB.2020.3040770, URL https: //ieeexplore.ieee.org/document/9314051/

Xiao F (2021b) GIQ: A Generalized Intelligent Quality-Based Approach for Fusing Multisource Information. IEEE Transactions on Fuzzy Systems 29(7):2018-2031. https://doi. org/10.1109/TFUZZ.2020.2991296

Xu H, Deng Y (2018) Dependent Evidence Combination Based on Shearman Coefficient and Pearson Coefficient. IEEE Access 6:11,63411,640. https://doi.org/10.1109/ACCESS.2017. 2783320

Xu Ly, Zhang Bf, Xu Ym, et al (2004) Evidence Ullage Analysis in D-S Theory and Development. JOURNAL OF SOFTWARE 15(1):69-75

Xu Pl, Yang Fb, Wang Xx, et al (2008) Study on Dempster-Shafer theory based on open frame of discernment. TRANSDUCER AND MICROSYSTEM TECHNOLOGIES 26(9):1113,9. https://doi.org/10.3969/j.issn.1000-9787. 2007.09.004

$\mathrm{Xu}$ Tx, Li Zq, Gu Jy, et al (2018) Missile Condition Assessment Based on Multistate Bayesian Network. Acta Armamentarii 39(2):391-398. https://doi.org/10.3969/j.issn. 1000-1093.2018.02.023

Xue D, Wang Y, Yang C (2021) An evidence combination approach based on fuzzy discounting.
Soft Comput 25(4):3003-3011. https://doi.org/ 10.1007/s00500-020-05359-9, URL https:/doi. org/10.1007/s00500-020-05359-9

Yager RR (1987) On the dempster-shafer framework and new combination rules. Information Sciences 41(2):93-137. https: //doi.org/10.1016/0020-0255(87)90007-7,

URL https://www.sciencedirect.com/science/ article/pii/0020025587900077

Ye F, Chen J, Li Y (2017) Improvement of DS Evidence Theory for Multi-Sensor Conflicting Information. Symmetry 9(5):69. https: //doi.org/10.3390/sym9050069, URL https:// www.mdpi.com/2073-8994/9/5/69

Zadeh LA (1984) Review of A Mathematical Theory of Evidence. AI Magazine 5(3):81-81. https://doi.org/10.1609/aimag.v5i3.452, URL https://ojs.aaai.org/index.php/aimagazine/ article/view/452

Zadeh LA (1986) A Simple View of the Dempster-Shafer Theory of Evidence and Its Implication for the Rule of Combination. AI Magazine 7(2):85-85. https://doi.org/ 10.1609/aimag.v7i2.542, URL https://ojs.aaai. org/index.php/aimagazine/article/view/542

Zhang H, Deng Y (2020) Weighted belief function of sensor data fusion in engine fault diagnosis. Soft Comput 24(3):2329-2339. https://doi.org/ 
10.1007/s00500-019-04063-7, URL https://doi. org/10.1007/s00500-019-04063-7

Zhang H, Lu JG, Tang XH (2020) An improved DS evidence theory algorithm for conflict evidence. Journal of Beijing University of Aeronautics and Astronautics 46(3):616-623. https: //doi.org/10.13700/j.bh.1001-5965.2019.0264

Zhao ZZ, Jia MP, He K, et al (2013) Approach of Combination of Weighted Evidence Based on Evidential Closeness Degree and its Application. Advanced Materials Research 630:377-382. https://doi.org/10. 4028/www.scientific.net/AMR.630.377, URL https://www.scientific.net/AMR.630.377

Zhou QL, Mo HM, Deng Y (2020) A New Divergence Measure of Pythagorean Fuzzy Sets Based on Belief Function and Its Application in Medical Diagnosis. Mathematics 8(1):142. https://doi.org/10.3390/math8010142, URL https://www.mdpi.com/2227-7390/8/1/142 